\title{
Effect of the S-PRG filler content in the multi-ion releasing paste on the acid resistance of the enamel surface after polishing with the paste
}

\author{
Koichi SHINKAI and Daiki YOSHII \\ Department of Operative Dentistry, The Nippon Dental University School of Life Dentistry at Niigata, 1-8 Hamaura-cho, Chuo-ku, Niigata 951-8580, \\ Japan \\ Corresponding author, Koichi SHINKAl; E-mail: shinkaik@ngt.ndu.ac.jp
}

\begin{abstract}
This study aimed to investigate the effect of the multi-ion releasing paste (MP) on the acid resistance of the enamel surface of an extracted human tooth. Five kinds of MP were prepared according to the content (wt\%) of S-PRG fillers: 0 wt\% (MP0, control), 1 wt\% (MP1), $5 \mathrm{wt} \%$ (MP5), $20 \mathrm{wt} \%$ (MP20), and $30 \mathrm{wt} \%$ (MP30). The buccal coronal surfaces of the extracted anterior teeth were polished with each kind of MP for 1 min. After removing radicular parts, the coronal parts underwent a $\mathrm{pH}$ cycling, and then sliced to make thin sections. The lesion depth of each section was measured using a polarization microscope. Each lesion's depth of enamel polished with MP5, MP20, and MP30 was significantly shallower than that polished with MP0.
\end{abstract}

Keywords: S-PRG filler, Multi-ion releasing paste, Acid resistance, Enamel surface, Polishing

\section{INTRODUCTION}

Previous studies have confirmed that dental caries occurs as a result of insufficient re-mineralization of the demineralized dental surface ${ }^{1-3)}$. Based on the minimal intervention (MI) concept for dental treatment ${ }^{4)}$, remineralization therapy, including fluoride application on the dental surface, professional mechanical tooth cleaning (PMTC), and dental home-care are implemented for early enamel caries without defects. PMTC is widely known as an effective preventive treatment for dental caries and periodontal disease. In PMTC, the polishing paste, which contains abrasive chemical agents such like fluoride, is rubbed against the dental surface using a rubber cup, with a slow rotary grinding instrument.

A surface reaction-type pre-reacted glass-ionomer (S-PRG) filler was developed to produce the stable glassionomer phase on the particles of fluoro-aluminosilicate glass using polyacrylic acid by the Shofu company (Kyoto, Japan). The S-PRG filler possesses ion-release and ion-recharge abilities similar to glass-ionomer cement $^{5-7)}$. Owing to this property, the S-PRG filler has been tried to utilize for various dental materials such as resin composite ${ }^{8,9)}$, dental sealant ${ }^{10-12)}$, tooth coating material ${ }^{13-15)}$, and direct pulp capping agents ${ }^{16,17}$.

Recently, the multi-ion releasing paste (MP) has been developed for dental surface polishing by the Shofu company. The MP contains multi-ion releasing abrasives and S-PRG fillers. When rubbing the MP against the dental surface, various ions such as aluminum (Al), boron $(\mathrm{B})$, fluoride $(\mathrm{F})$, sodium $(\mathrm{Na})$, silicon $(\mathrm{Si})$, and strontium ( $\mathrm{Sr}$ ) released from the S-PRG fillers may penetrate dental surface. Especially, Si, F, B, and Sr ions may contribute to increase the acid resistance of the dental surface due to their re-mineralization effect. Several studies have investigated the effects of toothpaste containing S-PRG fillers on the characteristics of tooth substance, and their results demonstrated the effectiveness of S-PRG fillers ${ }^{18-21}$. A few studies examined the effects of polishing with the MP on the acid resistance of the enamel surface; however, the available information is not enough ${ }^{21}$.

This study aimed to investigate the effect of MP on the acid resistance of the enamel surface of extracted human teeth. The null hypothesis was that polishing with MP would not influence the acid resistance of the enamel surface.

\section{MATERIALS AND METHODS}

Table 1 shows the composition of the materials used in this study, including each content of S-PRG fillers contained in the MP for the 5 experimental groups. The extracted human anterior teeth were used to examine the acid resistance of the enamel surface. Ethical approval for this study was obtained from the Nippon Dental University School of Life Dentistry at Niigata (ECNG-R-319).

\section{Preparation of the specimens}

The extracted human anterior teeth were stored in $0.1 \%$ thymol solution at $4^{\circ} \mathrm{C}$. The teeth for this study were selected using the following criteria: no cracks, no caries, no hypoplasia, and no application of hydrogen peroxide and fluoride. Thirty-five teeth were divided into 5 groups of 7 each. In each group, six teeth were used for the demineralization/re-mineralization test ( $\mathrm{pH}$-cycling test), and one for scanning electron microscope (SEM) observation.

The surfaces of the teeth were scaled using an ultrasonic scaler to remove attachments. The whole buccal enamel surface was polished for 1 min using a rubber cup with the respective MPs for each group shown in Table 1. After polishing, the enamel surface 
Table 1 Materials used in the present study

\begin{tabular}{|c|c|c|c|c|}
\hline Material & Group code & Main composition & Lot no. & Manufacturer \\
\hline $\begin{array}{l}\text { MP containing } \\
1 \text { wt\% S-PRG filler }\end{array}$ & MP1 & \multirow{5}{*}{$\begin{array}{l}\text { Anhydrous silica, } \\
\text { Carboxy methylcellulose sodium, } \\
\text { Glycerol, Sorbitol, Flavor, } \\
\text { Sodium lauryl sulfate, } \\
\text { S-PRG filler, Water }\end{array}$} & S-PRG69 & \multirow{5}{*}{ Shofu } \\
\hline $\begin{array}{l}\text { MP containing } \\
5 \mathrm{wt} \% \mathrm{~S}-\mathrm{PRG} \text { filler }\end{array}$ & MP5 & & S-PRG70 & \\
\hline $\begin{array}{l}\text { MP containing } \\
20 \text { wt } \% \text { S-PRG filler }\end{array}$ & MP20 & & S-PRG71 & \\
\hline $\begin{array}{l}\text { MP containing } \\
30 \mathrm{wt} \% \mathrm{~S}-\mathrm{PRG} \text { filler }\end{array}$ & MP30 & & S-PRG72 & \\
\hline $\begin{array}{l}\text { MP containing } \\
0 \text { wt\% S-PRG filler }\end{array}$ & $\begin{array}{l}\text { MP0 } \\
\text { (control) }\end{array}$ & & S-PRG66 & \\
\hline
\end{tabular}

MP: Multi-ion releasing paste, S-PRG filler: Surface reaction type Pre-reacted glass-ionomer filler based on fluoroboroaluminosilicate Glass

was sprayed for $5 \mathrm{~s}$ to sufficiently remove the MP. The specimens were then kept in distilled water at $37^{\circ} \mathrm{C}$.

\section{Demineralization/re-mineralization test}

After removing the root, a masking tape $(2 \times 2 \mathrm{~mm})$ was attached at the central area of the dental crown surface, and then, the all surface of the crown, including cut surface, was double-coated using nail varnish. After the coating material dried up, the masking tape was peeled off to expose the enamel surface polished with MP. The crowns coated with nail varnish, except the small enamel window, were used as specimens for the $\mathrm{pH}$-cycling test. The procedure of the $\mathrm{pH}$-cycling test used in this study was designed based on a previous study ${ }^{22}$

Six specimens for each group underwent $\mathrm{pH}$ cycling for 7 cycles. During the process of $\mathrm{pH}$ cycling, the specimens were immersed in a demineralizing solution for $18 \mathrm{~h}$, rinsed with distilled water for $5 \mathrm{~min}$, immersed in a re-mineralizing solution for $6 \mathrm{~h}$, and then rinsed with distilled water for $5 \mathrm{~min}$ again. The solutions were maintained at $37^{\circ} \mathrm{C}$ and stirred at $120 \mathrm{rpm}$ during $\mathrm{pH}$ cycling. The demineralizing solution was composed of $0.05 \mathrm{M}$ acetic acid, $2.2 \mathrm{mM}$ calcium ions, and $2.2 \mathrm{mM}$ phosphate ions ( $\mathrm{pH} 4.8$ ). The re-mineralizing solution was composed of $0.15 \mathrm{M}$ potassium chloride, $1.5 \mathrm{mM}$ calcium ions, and $0.9 \mathrm{mM}$ phosphate ions ( $\mathrm{pH} 7.0)$.

\section{Lesion-depth evaluation}

The enamel windows on the specimens were protected by the application of translucent nail varnish, after which the specimens were perpendicularly sectioned at the tooth axis through each enamel window using a hard-tissue microtome (Isomet, Buehler, Lake Bluff, IL, USA). Three sections with a thickness of approximately $200 \mu \mathrm{m}$ were obtained from each window. Each section was ground to a thickness of approximately $100 \mu \mathrm{m}$ using a lapping film sheet.

The sections were examined at a magnification of 200× using a polarized light microscope (PLM, Eclipse LV100POL, Nikon, Tokyo, Japan). Digital photomicrographs were obtained using a CCD camera
(DS-L2, Nikon). The lesion depth in each section was determined by measuring the distance between the original enamel surface and the deepest position of the lesion, using the camera's control software.

\section{SEM observation}

One specimen from each group was trimmed into two small cubes (approximately $2 \times 2 \times 2 \mathrm{~mm}$ ) containing the surface polished with MP, using a hard-tissue microtome (Isomet, Buehlar). The enamel surface of each cube was sputter-coated with $\mathrm{Au}-\mathrm{Pd}$, then observed in detail using SEM (S-800, Hitachi, Tokyo, Japan) at an accelerating voltage of $15 \mathrm{kV}$.

Component analysis of enamel surface using electron probe micro analyzer (EPMA)

One extracted human anterior tooth for each group was used for detecting the uptake of various ions by the flattened enamel surface. The buccal enamel surfaces of the anterior teeth were flattened and polished using a \#2000 silicon carbide paper (SANKYO RIKAGAKU, Saitama, Japan). Firstly, the half buccal enamel surface of the cervical side was covered with masking tape, and then the enamel surface of the other side was polished for $1 \mathrm{~min}$ with the respective MPs for each group, using a rubber cup. After cleaning the residual paste by spraying water and air-drying, the enamel surface was sputter-coated with carbon. The interface between the polished and unpolished sides was longitudinally probed for $2 \mathrm{~mm}$ using the EPMA (EPMA-1610, SHIMADZU, Kyoto, Japan).

\section{Statistical analysis}

The data of the lesion depth were statistically analyzed using the one-way ANOVA test, followed by Tukey's post-hoc test to compare the lesion depths between the five groups at a $95 \%$ confidence level. If the significant difference of variance among experimental groups were detected by Bartlett's test, the Kruskal-Wallis test, followed by the Steel Dwass post-hoc test, was applied. Statistical analysis was carried out using an add-in 
software package for Microsoft Excel (Bell Curve for Excel, Social Survey Research Information, Tokyo, Japan).

\section{RESULTS}

The means with SD of the lesion depths of experimental groups are shown in Fig. 1. The Kruskal-Wallis test, followed by Steel Dwass post-hoc test, were used for statistical analysis due to unequal variances. The results of the statistical analysis revealed that the lesion depths of MP5, MP20, and MP30 were significantly lower than that of MP0 (control) $(p<0.013)$, while those of MP20 and MP30 were significantly lower than that of MP1 $(p<0.002)$. There were no significant differences in lesion depth between MP0 and MP1, MP5 and MP20, or MP20 and MP30 $(p>0.05)$. The lesion depth of the enamel surface after $\mathrm{pH}$ cycling showed a tendency to decrease

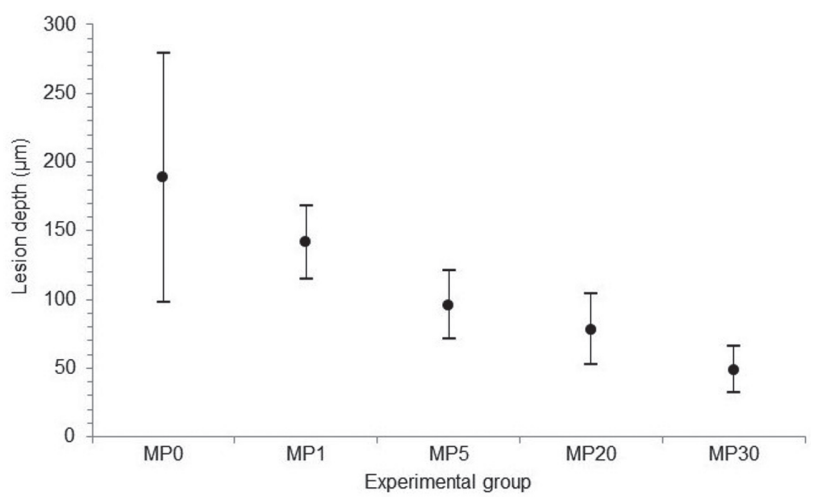

Fig. 1 Mean and SD values of lesion depth in each experimental group.
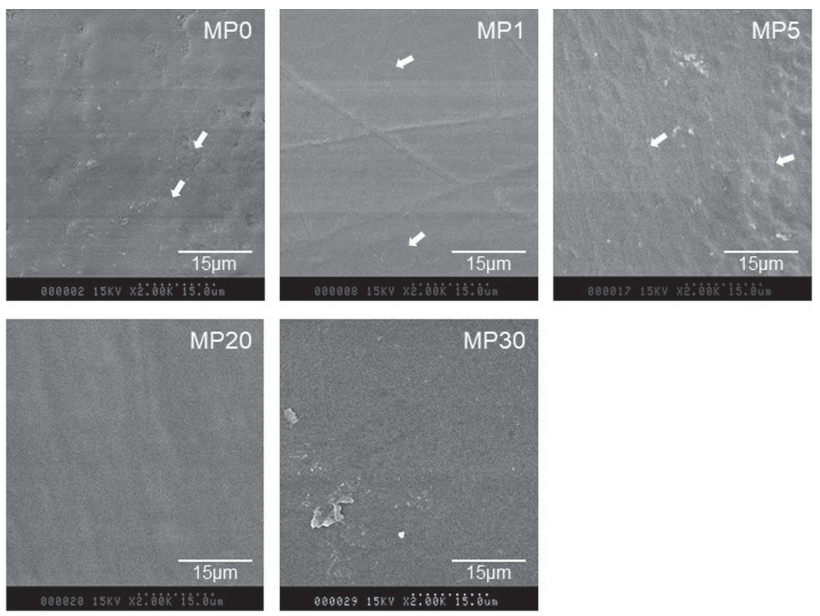

Fig. 2 SEM images of specimens after $\mathrm{pH}$ cycling in each experimental group.

MP20 and MP30 showed an amorphous texture of the enamel surfaces, while MP5, MP1, and MP0 showed a honeycomb-like structure. White arrows show honeycomb-like structures. Original magnification $(2,000 \times)$
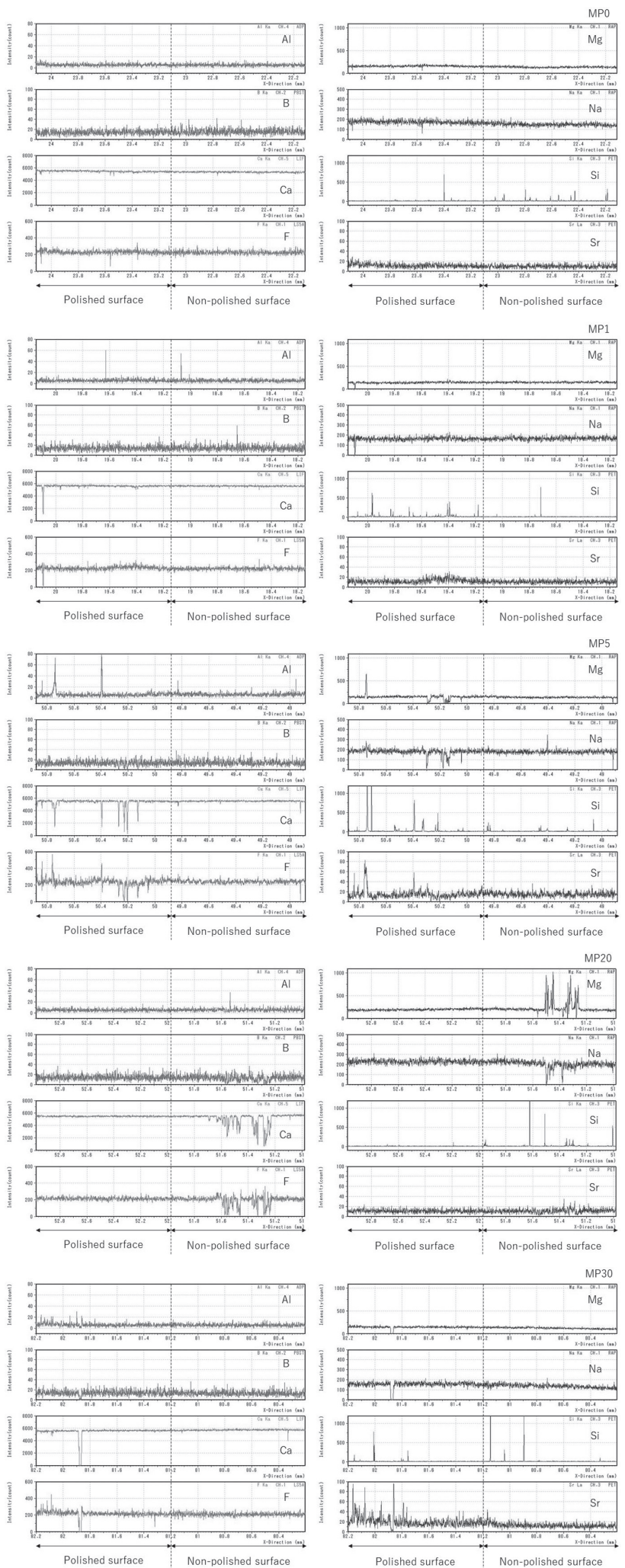

Fig. 3 EPMA analyses of the enamel surface polished with the respective MPs for each group.

The concentration of $\mathrm{Sr}^{2+}$ on the enamel surfaces polished with MP30 was slightly higher than that on the unpolished enamel surfaces. 
with increasing content of S-PRG fillers.

Representative SEM images are shown in Fig. 2. The SEM images of MP20 and MP30 specimens showed an amorphous texture of the enamel surfaces, and no significant difference was detected between them. On the SEM image of the other groups, MP5, MP1, and MP0 showed a honeycomb-like structure with an unclear line, that with a clear line, and that with some pores, respectively.

The results of the elemental analysis of the enamel surface using EPMA are shown in Fig. 3. The intensities of $\mathrm{B}, \mathrm{F}, \mathrm{Ca}, \mathrm{Si}, \mathrm{Mg}$ and $\mathrm{Na}$ ions on the enamel surfaces polished with MPs were almost same as those of the unpolished enamel surfaces in all groups. Meanwhile, the intensity of $\mathrm{Sr}$ ion on the enamel surfaces polished with MP30 was slightly higher than that of the unpolished enamel surfaces.

\section{DISCUSSION}

According to the results of this study, polishing with MP containing S-PRG fillers of more than $5 \mathrm{wt} \%$ were effective in increasing the acid resistance of the enamel surface, and the effectiveness was dependent on the wt\% of S-PRG fillers in the MP. Hence, the null hypothesis was rejected.

The SD of lesion depths of enamel surfaces polished with MP0 (control) was bigger than that of other MPs containing S-PRG fillers. The extracted teeth used in this study were obtained from a wide range of age groups. He et al. reported that the mineral densities of $\mathrm{Ca}$ and $\mathrm{P}$ in the outer layer of enamel in the old age group were significantly higher than those in the young age group $^{23)}$. Hence, the degree of calcification of the enamel surface may increase with the increasing age due to the absorption of mineral ions from surrounding substances by the enamel surface. Therefore, the difference in the degree of calcification of the extracted teeth might cause a wide range of lesion depths of the control without MP polishing.

In this study, the enamel surfaces after polishing with MP containing 20\% and 30\% S-PRG filler showed a significantly higher acid resistance than that with MP containing 1\% S-PRG filler. Iijima et al. examined the application of pastes containing various concentration of S-PRG fillers (0, 5, and $30 \mathrm{wt} \%)$ on enamel demineralization using human enamel blocks ${ }^{21)}$. The specimens polished with the pastes containing S-PRG filler for $10 \mathrm{~s}$ exhibited significantly more hardness and elastic modulus values than those polished with the tooth-brushing pastes without S-PRG fillers. These results indicated the tooth-brushing pastes containing S-PRG fillers inhibited the demineralization of the enamel surface, which is in line with the results of the present study. Amaechi et al. investigated the effect of S-PRG filler-containing toothpaste in various contents of the S-PRG filler on the inhibition of the demineralization of human the enamel surface, and showed that the toothpaste containing $20 \%$ and $30 \%$ S-PRG filler exhibited significantly less mineral loss than that containing $1 \% \mathrm{~S}-\mathrm{PRG}$ filler ${ }^{19)}$. The results of their study are similar to those of our study, although the procedures of application of toothpaste were different. They immersed the samples in a solution of the toothpaste dissolved in distilled water for $2 \mathrm{~min}$, while in our study, the surface of the samples was rubbed with the MP paste for $1 \mathrm{~min}$. Considering the clinical situation, the method of application used in this study might have been be more appropriate.

The SEM images of MP0 and MP1 specimens showed a visible honeycomb-like structure (enamel prisms), whereas those of MP20 and MP30 specimens had an amorphous texture and no visible prism outline. These findings suggested that the enamel surface of MP20 and MP30 specimens might resist acid attack during $\mathrm{pH}$ cycles, while specimens of other groups might lack resistance to acid attack. Hence, the effect of MP polishing on the demineralization of the enamel surface appears to depend on the concentration of the S-PRG filler, which corresponds to the results of the lesion-depth evaluation in the present study. Iijima reported that the specimens polished with $0 \% \mathrm{~S}-\mathrm{PRG}$ filler-containing paste exhibited very porous surfaces, with numerous visible prisms, whereas the specimens polished with 5 and 30\% S-PRG filler-containing pastes exhibited significantly smoother surfaces upon SEM observation, suggesting that the multiple ions released from the pastes improved the buffering capability of the enamel surfaces ${ }^{21)}$.

The efficacy of the MP containing S-PRG fillers in increasing the acid resistance of enamel shown in this study was supported by the results of previous studies which had demonstrated S-PRG filler-containing solutions to inhibit the demineralization of tooth substance ${ }^{24-27)}$. The multiple ions $\left(\mathrm{Sr}^{2+}, \mathrm{Na}^{+}, \mathrm{BO}_{3}{ }^{3-}\right.$, $\mathrm{Al}^{3+}, \mathrm{SiO}_{3}{ }^{2-}$, and $\mathrm{F}^{2-}$ ) eluted from $\mathrm{S}-\mathrm{PRG}$ fillers to the MP paste must be attributed to the increasing acid resistance of enamel. $\mathrm{SiO}_{3}{ }^{2-}$ is involved in the formation of hydroxyapatite under the existence of hydrated silica gel ${ }^{26)}$. Also, $\mathrm{SiO}_{3}{ }^{2-}$ and $\mathrm{Na}^{+}$lead to a buffering effect for the surrounding environment ${ }^{10,25)}$. Moreover, the simultaneous presence of $\mathrm{Sr}^{2+}$ with $\mathrm{F}^{2-}$ at specific concentrations enhanced enamel re-mineralization in vitro $^{27-29)}$. Therefore, it is speculated that the uptake of these multiple ions, especially $\mathrm{SiO}_{3}{ }^{2-}, \mathrm{Sr}^{2+}$, and $\mathrm{F}^{2-}$ to the enamel surface might arise during dental surface polishing with MP pastes; as such, the acid resistance of the enamel surface could increase.

The result of the EPMA analysis of the enamel surface polished with MP30 showed a slightly higher intensity of $\mathrm{Sr}^{2+}$ on the polished surface than on the unpolished surface. This result might have been due to the abundant supply of $\mathrm{Sr}^{2+}$ from MP30 to the ordinary enamel surface where Sr ions are of considerably lower intensity. While, the EPMA analysis for other groups did not show increasing the intensity of $\mathrm{Sr}^{2+}$ on the polished surface. The study, which dealt with various concentrations of S-PRG filler suspension, clarified the accumulation of $\mathrm{Sr}^{2+}$ on the bovine enamel surface immersed in a suspension of $10 \mathrm{wt} \% \mathrm{~S}-\mathrm{PRG}$ filler paste 
by means of particle-induced X-ray emission (PIXE) analysis $^{30)}$. Although the EPMA analysis carried out in the present study could not prove the accumulation of $\mathrm{Sr}^{2+}$ on the enamel surface polished with the polishing pastes including S-PRG fillers under $20 \mathrm{wt} \%$, the accumulated $\mathrm{Sr}^{2+}$ on the enamel polished with MP30 might infiltrate and form a $\mathrm{Ca}-\mathrm{Sr}$ apatite complex. Whereas, the concentration of the other ions, such as $\mathrm{Al}^{3+}, \mathrm{BO}_{3}{ }^{3-}, \mathrm{SiO}_{3}{ }^{2-}$, and $\mathrm{F}^{2-}$, were almost same on both polished and unpolished surfaces in all groups. Since these ions are in high concentrations on ordinary enamel surfaces, the supply of these ions from MP may be not clearly detected by EPMA analyses.

The S-PRG fillers might be stuck on the enamel surface when rubbing the MP using a rubber cup with rotation. From the stuck S-PRG fillers, various ions might infiltrate the superficial enamel surface. We tried to detect the stuck fillers on the enamel surface of specimens through SEM observation and EPMA analysis; however, no evidence for the existence of the stuck fillers was obtained. The evidence of this matter should be obtained by other methods in future studies.

\section{CONCLUSION}

Tooth polishing with MP containing S-PRG fillers of more than 5 wt\% exhibited a significantly higher acid resistance of the enamel surface than that of the paste without the S-PRG filler (control).

\section{ACKNOWLEDGMENTS}

This work was partially supported by Center for Coordination of Research Facilities (CCRF), Niigata University. The authors thank Ms. Ayako Ikarashi for her technical support for the EPMA analysis.

The authors deny any conflicts of interest related to this study.

\section{REFERENCES}

1) ten Cate JM, Duijsters PP. Alternating demineralization and remineralization of artificial enamel lesions. Caries Res 1982; 16: $201-210$

2) Featherstone JD, Glena R, Shariati M, Shields CP. Dependence of in vitro demineralization and remineralization of dental enamel on fluoride concentration. J Dent Res 1990; 69: $620-625$.

3) Featherstone JDB. The continuum of dental caries - Evidence for a dynamic disease process. J Dent Res 2004; 83(Spec Iss C): $\mathrm{C} 39-\mathrm{C} 42$

4) Tyas MJ, Anusavice KJ, Frencken JE, Mount GJ. Minimal intervention dentistry - a review. FDI commission project 1-97. Int Dent J 2000; 50: 1-12.

5) Ikemura K, Tay FR, Kouro Y, Endo T, Yoshiyama M, Miyai $\mathrm{K}$, et al. Optimizing filler content in an adhesive system containing pre-reacted glassionomer fillers. Dent Mater 2003; 19: $137-146$.

6) Fujimoto Y, Iwasa M, Murayama R, Miyazaki M, Nagafuji A, Nakatsuka T. Detection of ions released from S-PRG fillers and their modulation effect. Dent Mater J 2010; 29: 392-397.

7) Ogawa A, Wada T, Mori Y, Uo M. Time dependence of multiion absorption into human enamel from surface prereacted glass-ionomer (S-PRG) filler eluate. Dent Mater J 2019; 38: 707-712.

8) Miki S, Kitagawa H, Kitagawa R, Kiba W, Hayashi M, Imazato S. Antibacterial activity of resin composites containing surface pre-reacted glass-ionomer (S-PRG) filler. Dent Mater 2016; 32: 1095-1102

9) Kitagawa H, Miki-Oka S, Mayanagi G, Abiko Y, Takahashi $\mathrm{N}$, Imazato S. Inhibitory effect of resin composite containing S-PRG filler on Streptococcus mutans glucose metabolism. J Dent 2018; 70: 92-96.

10) Kaga M, Kakuda S, Ida Y, Toshima H, Hashimoto M, Endo $\mathrm{K}$, et al. Inhibition of enamel demineralization by buffering effect of S-PRG filler-containing dental sealant. Eur J Oral Sci 2014; 122: 78-83

11) Shimazu K, Ogata K, Karibe H. Caries-preventive effect of fissure sealant containing surface reaction-type pre-reacted glass ionomer filler and bonded by self-etching primer. J Clin Pediatr Dent 2012; 36: 343-347.

12) Ntaoutidou S, Arhakis A, Tolidis K, Kotsanos N. Clinical evaluation of a surface pre-reacted glass (S-PRG) fillercontaining dental sealant placed with a self-etching primer/ adhesive. Eur Arch Paediatr Dent 2018; 19: 431-437.

13) Kawasaki K, Kambara M. Effects of ion-releasing toothcoating material on demineralization of bovine tooth enamel. Int J Dent 2014; 463149.

14) Okuyama K, Kadowaki Y, Matsuda Y, Hashimoto N, Oki S, Yamamoto $\mathrm{H}$, et al. Efficacy of a new filler-containing root coating material for dentin remineralization. Am J Dent 2016; 29: 213-218.

15) Shiiya T, Mukai $Y$, Tomiyama K, Teranaka T. Antidemineralization effect of a novel fluoride-releasing varnish on dentin. Am J Dent 2012; 25: 347-350.

16) Kawashima S, Shinkai K, Suzuki M. Effect of an experimental adhesive resin containing multi-ion releasing fillers on direct pulp-capping. Dent Mater J 2016; 35: 479-489.

17) Takahashi Y, Okamoto M, Komichi S, Imazato S, Nakatsuka $\mathrm{T}$, Sakamoto S, et al. Application of a direct pulp capping cement containing S-PRG filler. Clin Oral Investig 2019; 23: 1723-1731.

18) Amaechi BT, Key MC, Balu S, Okoye LO, Gakunga PT. Evaluation of the caries-preventive effect of toothpaste containing surface prereacted glass-ionomer filler. J Investig Clin Dent 2017; 8. doi: 10.1111/jicd.12249.

19) Amaechi BT, Kasundra H, Joshi D, Abdollahi A, Parveez AAA, Okoye LO. Effectiveness of S-PRG filler-containing toothpaste in inhibiting demineralization of human tooth surface. Open Dent J 2018; 12: 811-819.

20) Tomiyama K, Shiiya T, Watanabe K, Hamada N, Mukai Y. Effect of toothpaste containing multiple ions-releasing filler on polymicrobial biofilm regrowth and dentin demineralization. Am J Dent 2019; 32: 245-250.

21) Iijima M, Ito S, Nakagaki S, Kohda N, Muguruma T, Saito $\mathrm{T}$, et al. Effects of immersion in solution of an experimental toothpaste containing S-PRG filler on like-remineralizing ability of etched enamel. Dent Mater J 2014; 33: 430-436.

22) Gao XL, Pan JS, Hsu CY. Laser-fluoride effect on root demineralization. J Dent Res 2006; 85: 919-923.

23) He B, Huang S, Zhang C, Jing J, Hao Y, Xiao L, et al. Mineral densities and elemental content in different layers of healthy human enamel with varying teeth age. Arch Oral Biol 2011; 56: 997-1004.

24) Saito T, Toyooka H, Ito S, Crenshaw MA. In vitro study of remineralization of dentin: effects of ions on mineral induction by decalcified dentin matrix. Caries Res 2003; 37: 445-449.

25) Czarnecka B, Limanowska-Shaw H, Nicholson JW. Buffering and ion-release by a glass-ionomer cement under near-neutral and acidic conditions. Biomaterials 2002; 23: 2783-2788.

26) Asano $K$, Kawamoto $R$, Iino $M$, Fruichi $T$, Nojiri $K$, Takamizawa T, et al. Effect of pre-reacted glass-ionomer filler 
extraction solution on demineralization of bovine enamel. Oper Dent 2014; 39: 159-165.

27) Featherstone JD, Shields CP, Khademazad B, Oldershaw MD. Acid reactivity of carbonated apatite with strontium and fluoride substitutions. J Dent Res 1983; 62: 1049-1053.

28) Thuy TT, Nakagaki H, Kato K, Hung PA, Inukai J, Tsuboi $\mathrm{S}$, et al. Effect of strontium in combination with fluoride on enamel remineralization in vitro. Arch Oral Biol 2008; 53: 1017-1022.
29) Yassen GH, Lippert F, Eckert G, Eder J, Zandoná AF. The effect of strontium and combinations of strontium and fluoride on the remineralization of artificial caries lesions in vitro. Quintessence Int 2012; 43: e95-103.

30) Nakamura K, Hamba H, Nakashima S, Sadr A, Nikaido T, Oikawa M, et al. Effects of experimental pastes containing surface pre-reacted glass ionomer fillers on inhibition of enamel demineralization. Dent Mater J 2017; 36: 482-490. 